\title{
Análise morfométrica e de rendimento em carne de Mytilidae capturado no estado de Sergipe
}

Morphometric analysis and meat yield of Mytilidae caught in the state of Sergipe

\author{
J. J. C. Reis Júnior ${ }^{1 *}$; K. M. F. Freire ${ }^{1}$; L. C. Rosa ${ }^{2}$; A. C. G. Santos ${ }^{1}$; A. Silva \\ Lúcio $^{3}$; B. S. Santiago²; B. V. Santos ${ }^{3}$; I. S. Silva 3 ; J. V. Bispo ${ }^{3}$; L. S. Rocha $;$ M. \\ C. S. Freire ${ }^{3}$; R. T. V. S. Santos 3 ; R. C. D. Lima ${ }^{3}$; S. L. Santos ${ }^{1}$ \\ ${ }^{1}$ Departamento de Engenharia de Pesca e Aquicultura (DEPAQ)/Laboratório de Ecologia Pesqueira (LEP), \\ Universidade Federal de Sergipe (UFS), 49100-000, São Cristóvão-SE, Brasil \\ ${ }^{2}$ Departamento de Engenharia de Pesca e Aquicultura (DEPAQ)/Laboratório de Ecologia Bentônica, Universidade \\ Federal de Sergipe (UFS), 49100-000, São Cristóvão-SE, Brasil \\ ${ }^{3}$ Curso de Graduação em Engenharia de Pesca, Universidade Federal de Sergipe (UFS), 49100-000, São Cristóvão- \\ SE, Brasil \\ *josafajunior13@hotmail.com
}

(Recebido em 05 de abril de 2016; aceito em 11 de outubro de 2016)

\begin{abstract}
Duas espécies de Mytella ocorrem no estado de Sergipe, M. charruana e M. bicolor, sendo ambas explotadas, porém em proporção desconhecida. Um dos primeiros passos para se avaliar corretamente a importância de cada uma das espécies na economia local é avaliar o rendimento em carne. Assim, o presente trabalho foi concebido com o objetivo de avaliar o rendimento em carne e ao mesmo tempo as relações morfométricas de ambas as espécies. Foram coletadas amostras mensais de aproximadamente $2 \mathrm{~kg}$ para cada espécie nos meses de abril a junho de 2014. As relações entre a largura (LC) e o comprimento (CC) da concha estimadas para $M$. charruana e M. bicolor foram LC $=3,7965+0,3391 \mathrm{CC}$ e $\mathrm{LC}=5,1308+$ 0,3934 CC, respectivamente. As relações entre a altura (AC) e o comprimento (CC) da concha foram AC $=-1,9163+0,3546 \mathrm{CC}$ e AC $=1,1267+0,2977 \mathrm{CC}$, respectivamente. Finalmente, as relações entre o peso total $(\mathrm{PT})$ e o comprimento da concha $(\mathrm{CC})$ foram $\mathrm{PT}=0,0004 \mathrm{CC}^{2,3976}$ e $\mathrm{PT}=8 \mathrm{E}-05 \mathrm{CC}^{2,8437}$, respectivamente. Em todos os casos, foi observada uma diferença estatisticamente significante na relação para ambas as espécies, havendo no geral uma alometria negativa. $\mathrm{O}$ rendimento médio de carne in natura para M. charruana e M. bicolor foi de 50,3 e 33,2\%, respectivamente. Dessa forma, para obter um quilo de carne (catado) seria preciso coletar aproximadamente $2 \mathrm{~kg}$ de indivíduos inteiros (carne e concha) de $M$. charruana e $3 \mathrm{~kg}$ de M. bicolor.

Palavras-chave: rendimento em carne, alometria, morfometria
\end{abstract}

Two species of Mytella occur in the state of Sergipe, M. charruana and M. bicolor. Both of them are exploited but in an unknown proportion. One of the first steps towards the proper assessment of the importance of each species for the local economy is to estimate their meat yield. Thus, this study was carried out with the objective of estimating the meat yield and the morphometric relationships for each of the exploited species. Monthly samples of about $2 \mathrm{~kg}$ were collected for each species from April to June 2014. The estimated relationships between shell width (SW) and length (SL) for M. charruana and M. bicolor were $\mathrm{SW}=3.7965+0.3391 \mathrm{SL}$ e SW $=5.1308+0.3934 \mathrm{SL}$, respectively. The relationships between shell height $(\mathrm{SH})$ and length $(\mathrm{SL})$ were $\mathrm{SH}=-1.9163+0.3546 \mathrm{SL}$ and $\mathrm{SH}=1.1267+0.2977 \mathrm{SL}$, respectively. Finally, the relationships between total weight (TW) and shell length (SL) were TW $=0.0004 \mathrm{SL}^{2.3976}$ and $\mathrm{TW}=8 \mathrm{E}-05 \mathrm{SL}^{2.8437}$, respectively. In all relationships, a statistically significant difference was observed between the two species studied, with a tendency towards negative allometry. The mean in natura meat yield for M. charruana and M. bicolor were 50.3 and 33.2\%, respectively. Thus, in order to obtain $1 \mathrm{~kg}$ of meat ('catado'), about $2 \mathrm{~kg}$ and $3 \mathrm{~kg}$ of whole specimens (meat and shell) of M. charruana and M. bicolor are required, respectively.

Keywords: meat yield, allometry, morphometry 


\section{INTRODUÇÃO}

O manguezal é um ecossistema fundamental na manutenção da qualidade de água, fixação do sedimento e fornecimento de produção primária para conservação da biodiversidade, sendo considerado berçário e área de refúgio para múltiplas espécies marinhas e estuarinas de interesse comercial, tornando-se um dos ambientes costeiros mais produtivos [1].

Associados a esse ambiente podemos encontrar os mexilhões da família Mytilidae de interesse comercial, destacando-se quatro gêneros: Brachidontes, Perna, Mytilus e Mytella [2]. Esses bivalves são empregados em larga escala na alimentação humana, representando fonte de proteína animal de baixo custo e de alto valor nutricional [3]. Em Sergipe, podemos encontrar duas espécies de Mytilidae, as quais são conhecidas localmente como sururu: o sutinga (Mytella charruana) e o sururu de mangue (Mytella guyanensis = bicolor), sendo ambas as espécies explotadas, gerando renda para muitas famílias [4].

Mytella charruana é considerado sinônimo de Mytella falcata [5], uma espécie que possui uma ampla distribuição em estuários brasileiros, vivendo aderido aos substratos rochosos ou lamosos dos estuários por meio do bisso e formando colônias em regiões entre-marés com profundidade de até $10 \mathrm{~m}$. Esses bivalves estão adaptados a permanecerem expostos ao ar, ocorrendo nesse período queda do metabolismo, sem maiores danos ao organismo. O Mytella guyanensis, também conhecido como mexilhão de estuário, bacucu ou bico de ouro, é encontrado em bosques de mangue situados na zona entre-marés de ambientes estuarinos [6,7]. De acordo com Sartori \& Rosenberg (2016) [8], o nome M. guyanensis é sinônimo de Mytella bicolor, não sendo mais aceito o primeiro nome.

Araújo et al. (2009) [9] ressaltam a importância da pesca extrativa do sutinga no povoado de Taiçoca de Fora (Sergipe), onde mais de $90 \%$ da população desse povoado tem o marisco como fonte de renda, sendo possível observar, em quase todas as casas dos moradores, famílias inteiras separando o sutinga da concha. Dados do IBAMA (2007) [10] indicaram que, em Sergipe, a captura de sururu (Mytella falcata e Mytella spp., de acordo com aquela fonte) foi de 134,5 t no ano de 2007 , representando quase $100 \%$ da produção de moluscos no estado. Com relação à geração de renda, a pesca do sururu contribuiu com aproximadamente R $\$ 640 \mathrm{mil}$, ou seja, 3\% do valor total correspondente ao valor de primeira venda do pescado marinho-estuarino. Dados publicados pelo Projeto de Monitoramento Participativo de Desembarque Pesqueiro (PMPDP) indicam que a produção total de sururu (Mytella spp.) no estado de Sergipe variou de 32,0 a 60,1 t em 2010-2013, sendo os moluscos mais capturados no estado [11, 12, 13, 14]. Porém, não há registro separadamente da captura de cada uma das espécies de Mytella que ocorrem no estado.

O registro da produção do sururu é muitas vezes dificultado pelo fato de se registrar apenas a produção do seu catado (indivíduo sem a concha). Assim, faz-se necessário obter o rendimento em carne de cada espécie. Estudos sobre rendimento do sururu foram encontrados para $M$. guyanensis (=bicolor) no Paraná [15] e M. charruana em Alagoas [16], não sendo encontrado nenhum trabalho para o estado de Sergipe.

Estudos de morfometria são importantes a fim de possibilitar a comparação entre espécies do mesmo gênero e entre indivíduos da mesma espécie, mas de regiões diferentes, assim como para utilização em modelos de avaliação pesqueira [17]. Adicionalmente, são importantes para estimar o peso total de espécimes encontrados no estudo estomacal de predadores, a partir de pedaços de conchas que normalmente são os únicos remanescentes do item ingerido, como o caso dos otólitos e vértebras para peixes [18]. Embora alguns estudos tenham sido realizados sobre a reprodução e estrutura populacional de $M$. charruana e M. bicolor [7, 19, 20], nenhum trabalho foi encontrado que analisasse a morfometria das suas conchas.

Assim, o presente estudo teve como objetivo avaliar o rendimento em carne de M. charruana e $M$. bicolor capturados em Sergipe, visto que esses moluscos são um recurso de grande importância socioeconômica para o estado, além de analisar a morfometria dessas espécies.

\section{MATERIAL E MÉTODOS}

As amostras de $M$. charruana foram coletadas no estuário do Rio do Sal, no povoado de Taiçoca de Fora, no município de Nossa Senhora do Socorro, e de Mytella bicolor, no estuário do 
Rio Cotinguiba, no município de Laranjeiras (Figura 1). Uma amostra de aproximadamente $2 \mathrm{~kg}$ de cada espécie foi coletada manualmente por um(a) pescador(a) por mês nos meses de abril a junho de 2014, durante a maré baixa de sizígia. A amostra foi devidamente etiquetada e acondicionada em freezer para posterior análise no Laboratório de Ecologia Pesqueira (LEP/DEPAQ/UFS).

A identificação das duas espécies de Mytella foi realizada de acordo com Rios (1985) [21]. Foram medidas as variáveis comprimento $(\mathrm{CC}, \mathrm{mm})$, largura $(\mathrm{LC}, \mathrm{mm})$ e altura da concha $(\mathrm{AC}$, $\mathrm{mm}$ ), utilizando um paquímetro com precisão de $1 \mathrm{~mm}$ (Figura 2). Foram também determinados o peso total (PT, g) e o peso da concha (PCo, g) utilizando uma balança digital com precisão de $0,01 \mathrm{~g}$. O peso da carne (PCa, g) foi calculado através da diferença entre o peso total e o peso da concha.

Foram estimadas relações lineares entre a largura e a altura e o comprimento da concha, a fim de avaliar a isometria no crescimento da concha $(\mathrm{LC}=\mathrm{a}+\mathrm{bCC}$ e $\mathrm{AC}=\mathrm{a}+\mathrm{bCC})$. As relações estimadas foram comparadas entre as duas espécies através do intervalo de confiança para os parâmetros a e b. Foram estimadas relações peso-comprimento para cada uma das espécies através do modelo potencial: $\mathrm{PT}=\mathrm{aCC}^{\mathrm{b}}$. As relações para as duas espécies foram comparadas através do intervalo de confiança para "a" e "b".

$O$ rendimento em peso de cada uma das espécies foi determinado como a percentagem entre o peso da carne e o peso total do exemplar (carne e concha), sendo avaliado por classes de comprimento, a fim de observar a existência de variação ao longo da vida da espécie.

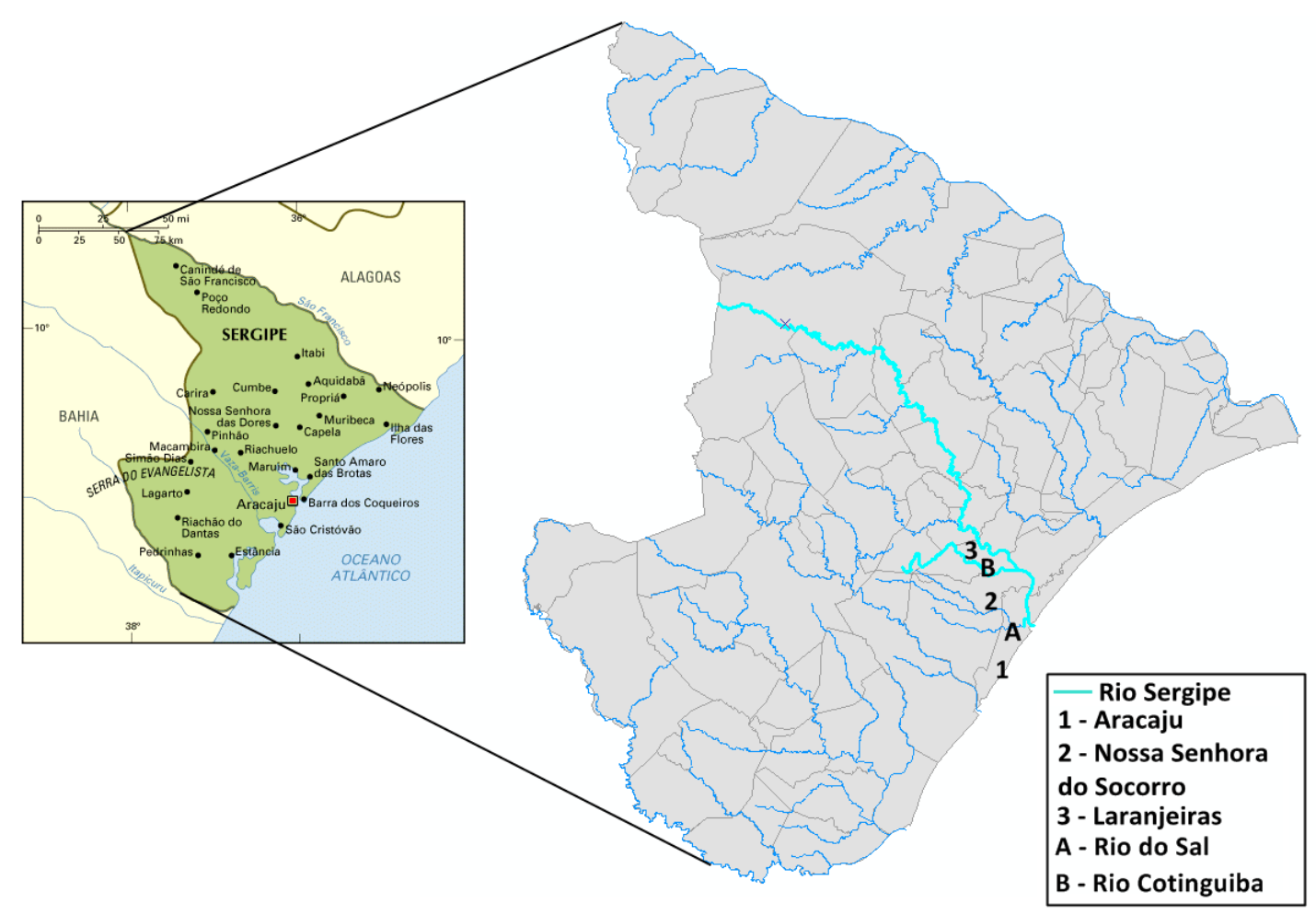

Figura 1: Mapa de Sergipe indicando os municípios de Aracaju (1), Nossa Senhora do Socorro (2)e Laranjeiras (3), o Rio Sergipe e dois dos seus afluentes: Rio do Sal (A) e Rio Cotinguiba (B), ambos pontos de amostragem desse trabalho. 

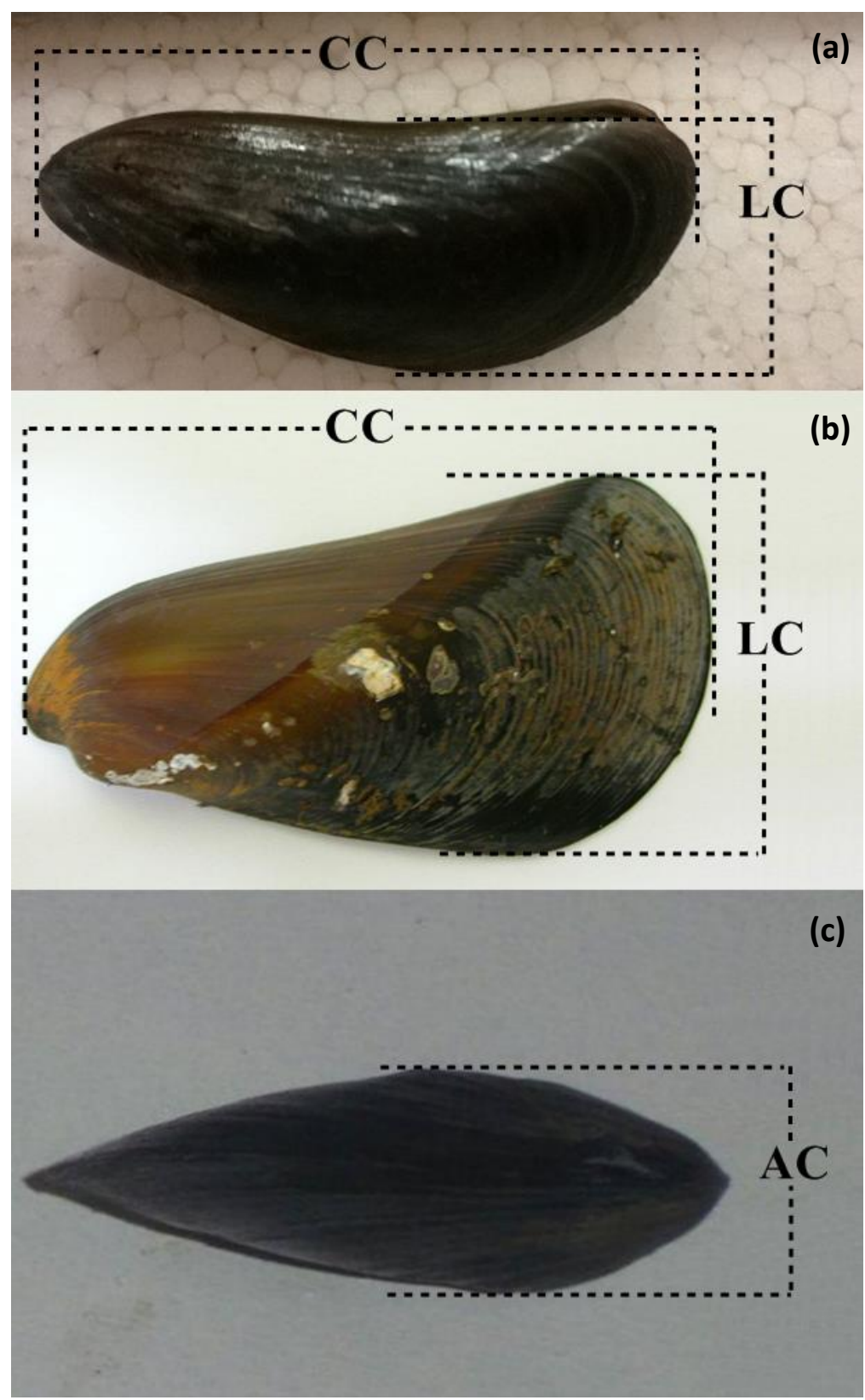

Figura 2: Comprimento (CC) e largura (LC) da concha para M. charruana (a) e M. bicolor (b); altura $(A C)$ da concha representada apenas para M. charruana (c) em Sergipe.

\section{RESULTADOS E DISCUSSÃO}

Foi observado que as amostras coletadas em bancos distintos, em dois estuários diferentes em Sergipe, raramente apresentaram as duas espécies juntas. Do mesmo modo, Pereira et al. (2003, 2007) [7, 22] observaram que os bancos naturais de $M$. charruana e $M$. bicolor raramente se misturavam entre si. No total, foram amostrados 435 exemplares de $M$. charruana e 300 de $M$. bicolor. A M. charruana apresentou um comprimento da concha que variou de 20 a $55 \mathrm{~mm}$ (39 $\pm 6,4$ ), sendo observada a presença de duas modas correspondentes a duas coortes (Figura 3). A M. bicolor teve um comprimento da concha de 31 a $63 \mathrm{~mm}(47 \pm 5,9)$, sendo observada apenas uma moda na distribuição de comprimento (Figura 3). 

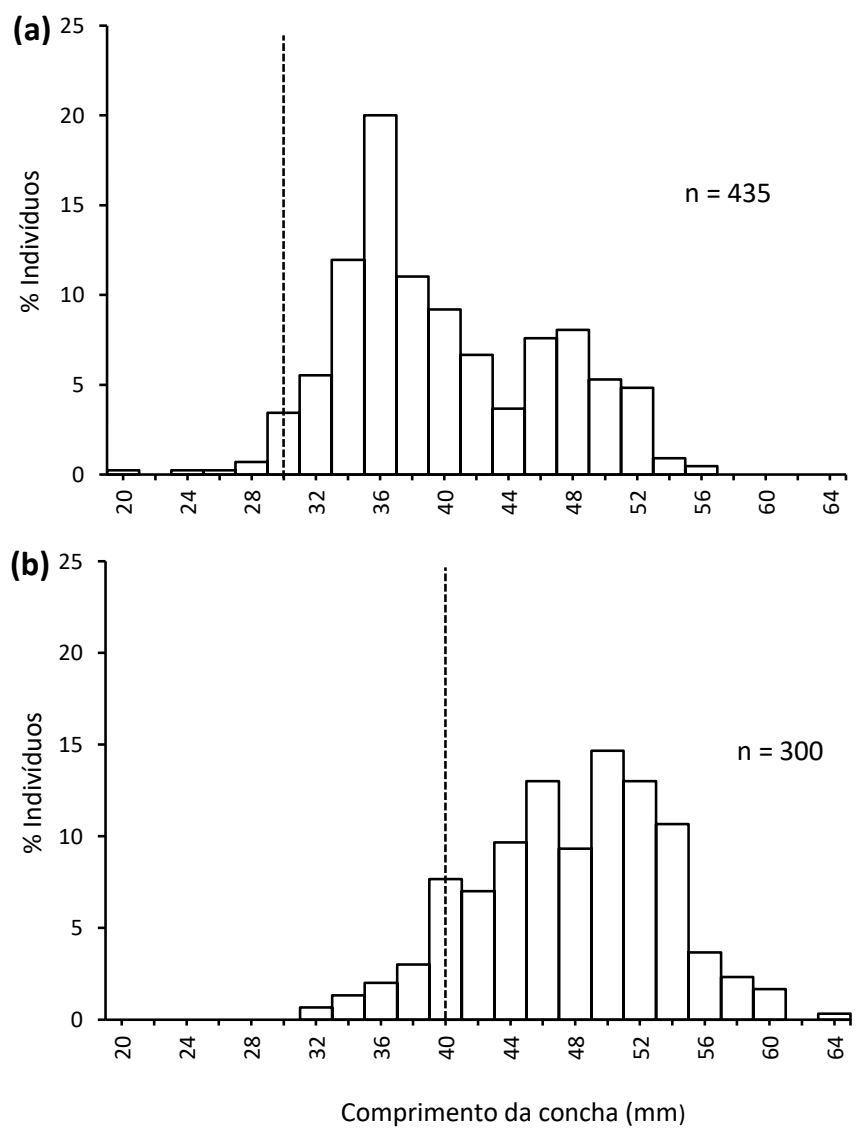

Figura 3: Distribuição de frequência de comprimento da concha (CC) para as espécies $\mathrm{M}$. charruana (a) e M. bicolor (b) capturadas em Sergipe. As linhas tracejadas indicam o tamanho de comercialização adotado em São Paulo de acordo com Pereira et al. (2003) [7].

M. charruana atingiu comprimento de concha inferior ao de M. bicolor. Porém, o comprimento máximo observado nesse estudo para $M$. charruana $(55 \mathrm{~mm})$ foi superior ao anteriormente encontrado por Araújo et al. (2009) [9] em Taiçoca de Fora, no estado de Sergipe (44 mm), assim como por Pereira et al. (2003) [7] para o estado de São Paulo (46 mm). O máximo comprimento da concha observado nesse estudo para M. bicolor $(63 \mathrm{~mm})$ foi um pouco inferior ao encontrado por Nishida \& Leonel (1995) [19] para o estado da Paraíba (65 mm) e por Pereira et al. (2003) [7] para o estado de São Paulo (65 mm), porém muito inferior ao máximo observado por CarpesPaternoster (2003) [23] para o estado de Santa Catarina $(86 \mathrm{~mm})$. Os resultados observados para as duas espécies não permitem observar o padrão usualmente aceito de aumento de comprimento com o aumento da latitude, exceto pelo valor extremamente elevado dos espécimes de Santa Catarina. Porém, deve-se considerar o efeito da taxa de explotação dos estoques sobre o comprimento máximo. Henriques et al. (2004) [24], por exemplo, analisaram o tamanho do comprimento da concha do mitilídeo Perna perna em bancos naturais da Baía de Santos (SP) e concluíram que os maiores tamanhos dos indivíduos foram encontrados nos bancos menos explotados. Por outro lado, Santos et al. (2010) [25] analisaram o comprimento da concha e o peso seco de $M$. charruana em dois bancos explotados e em um banco virgem, e observaram menores valores no banco virgem devido à baixa frequência com a qual eram inundados pela maré. Assim, outros fatores além do grau de explotação estão envolvidos.

As relações estimadas entre a largura (LC) e o comprimento (CC) da concha para M. charruana e M. bicolor foram $\mathrm{LC}=3,7965+0,3391 \mathrm{CC}$ e LC $=5,1308+0,3934 \mathrm{CC}$, respectivamente (Figura 4). As relações entre a altura (AC) e o comprimento (CC) da concha foram AC $=-1,9163$ $+0,3546 \mathrm{CC}$ e $\mathrm{AC}=1,1267+0,2977 \mathrm{CC}$, respectivamente (Figura 5). As relações entre o peso total e o comprimento da concha foram PT $=0,0004 \mathrm{CC}^{2,3976}$ e PT $=8 \mathrm{E}-05 \mathrm{CC}^{2,8437}$, respectivamente (Figura 6). Os intervalos de confiança calculados para os parâmetros "a" e "b" 
para todas as relações morfométricas estimadas (Tabela 1) indicaram diferença estatisticamente significante para as duas espécies.
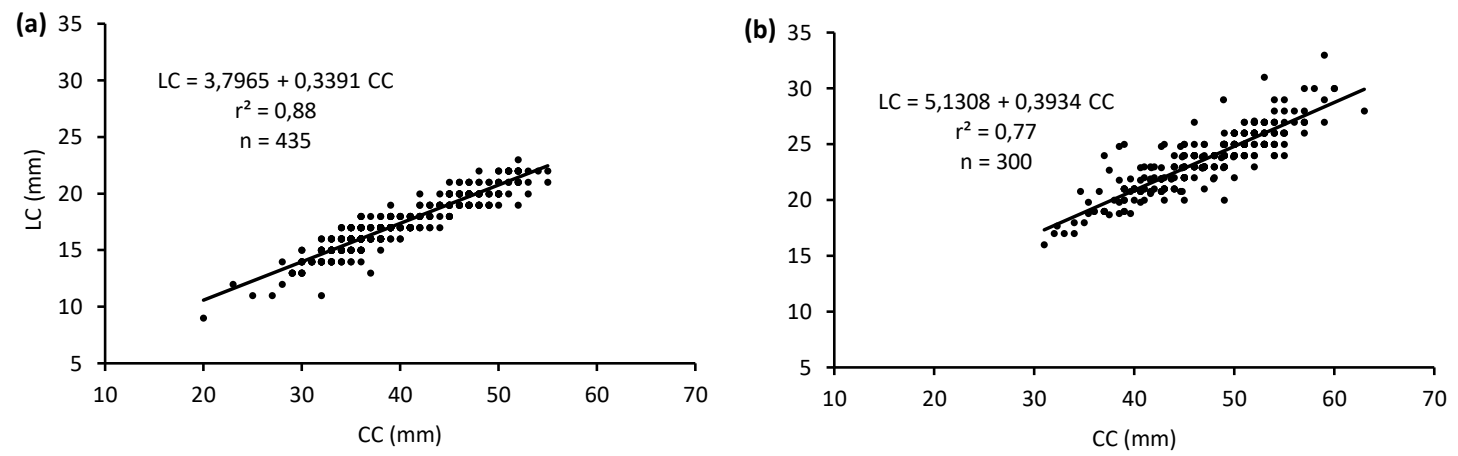

Figura 4: Relação entre a largura $(L C)$ e o comprimento $(C C)$ da concha para M. charruana (a) $e$ M. bicolor (b) em Sergipe.

(a)

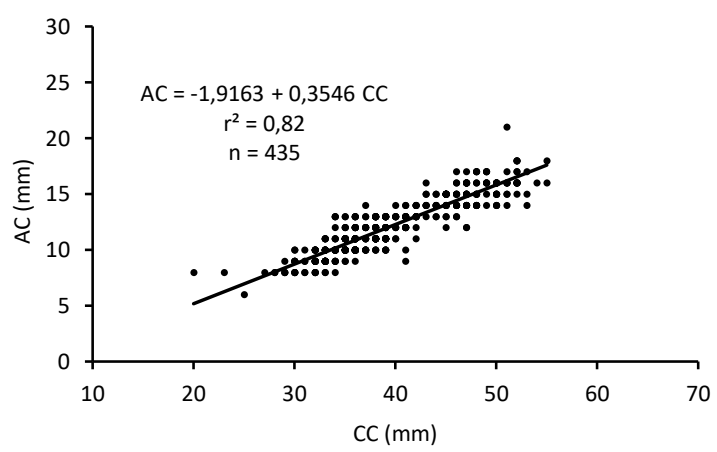

(b)

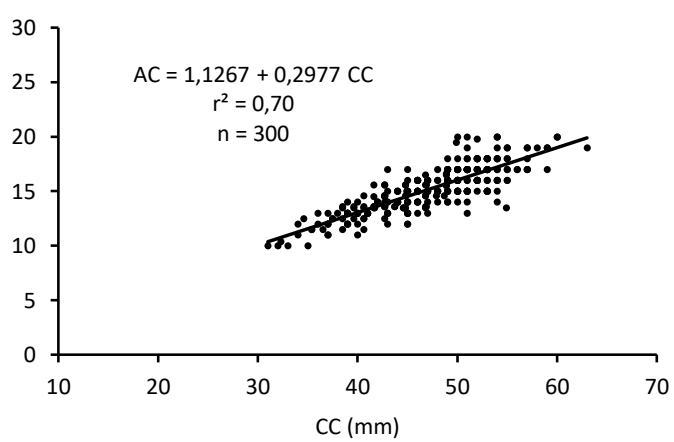

Figura 5: Relação entre a altura (AC) e o comprimento $(C C)$ da concha para $\mathrm{M}$. charruana $(a)$ e M. bicolor (b) em Sergipe.
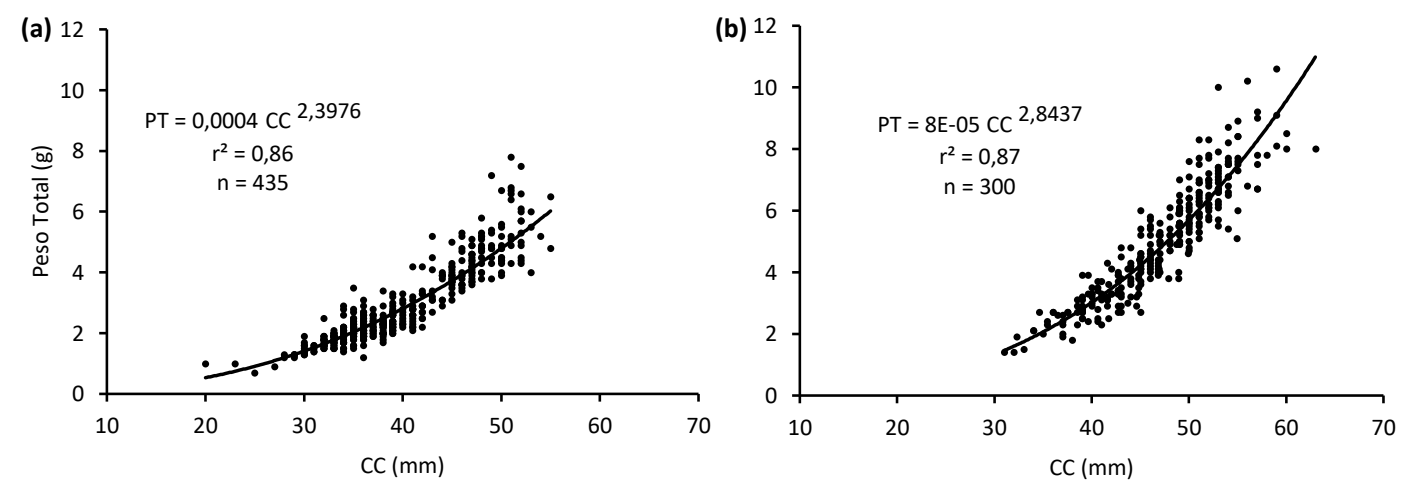

Figura 6: Relação entre o peso total e o comprimento da concha (CC) para M. charruana (a) $e$ M. bicolor (b) em Sergipe.

As relações comprimento-comprimento estimadas indicaram uma alometria negativa no crescimento, ou seja, o crescimento em largura e altura da concha acompanha proporcionalmente o crescimento em comprimento com uma proporção inferior a 1. Katsanevakis et al. (2006) [26] chamam a atenção para o cuidado que se deve ter ao analisar a alometria negativa uma vez que pode estar associada, por exemplo, a dados segmentados, implicando em mudança de declividade nas relações analisadas. Porém, esse não parece ser o caso para Mytella nesse trabalho. 
Ambas as espécies apresentaram crescimento alométrico negativo $(b<3)$ sensu Froese (2006) [27] quando analisada a relação entre o peso total e o comprimento da concha (Figura 5). Porém nota-se que, além de atingir maior comprimento, a M. bicolor apresenta maior peso para a mesma classe de comprimento, como evidenciado pelo valor de "b" superior $(2,8436)$. Apesar do valor reduzido de "b" observado para $M$. charruana, ele é muito superior ao valor de 1,90 encontrado por Araújo et al. (2009) [9] para o estuário do Rio Sergipe, também no estado de Sergipe. Porém, deve-se observar que os resultados apresentados naquele trabalho parecem indicar a existência de duas curvas possivelmente devido ao agrupamento das duas espécies de Mytella. Para outros moluscos, os resultados são variáveis. Yauri et al. (2011) [28], por exemplo, encontrou, no estado do Maranhão, espécies com crescimento alométrico negativo $(b<3)$ como a Anomalocardia brasiliana $(\mathrm{b}=1,47)$, Tagelus plebeius $(\mathrm{b}=2,44)$ e Macoma constricta $(\mathrm{b}=2,94)$, e com crescimento alométrico positivo (b>3), como Protothaca antiqua ( $b=3,17)$. Por outro lado, Schaeffer-Novelli (1980) [29] encontrou um alometria positiva $(b=3,23)$ para Anomalocardia brasiliana em Ubatuba (SP).

Arrieche et al. (2002) [30] encontraram uma alometria negativa $(b=2,47)$ para o mexilhão Perna perna na amostra total correspondente ao período de um ano. Porém, esses autores encontraram alguns meses com alometria positiva quando as amostras de cada mês foram tratadas separadamente. Apesar de diferenças entre condições ambientais afetarem o valor de "b" ao longo do ano, diferenças da magnitude observadas para A. brasiliana nos dois estudos citados não é esperada. Deve-se tomar cuidado, antes de mais nada, com os modelos efetivamente utilizados para a estimativa da relação peso-comprimento (geralmente o potencial), o modo como o comprimento foi medido e, adicionalmente, a ordem das variáveis consideradas (variável dependente e independente), a fim de que se possa comparar apenas resultados provenientes de modelos idênticos (ver, p.ex., diferenças observadas nos modelos apresentados em Souza (2012) [31] e Santos et al. (2014) [32], ambos para A. brasiliana).

O rendimento em carne (\%) se manteve aproximadamente constante para diferentes classes de comprimento da concha $(\mathrm{mm})$ em ambas as espécies (Tabela 2), sendo o rendimento médio de carne de M. charruana (50,3\%) superior ao de M. bicolor (33,2\%). Dessa forma, para obter um quilo de carne (catado in natura) seria preciso coletar aproximadamente $2 \mathrm{~kg}$ de conchas fechadas de $M$. charruana e $3 \mathrm{~kg}$ de $M$. bicolor. Nota-se que a maior parte dos exemplares amostrados de M. charruana se encontravam acima dos $30 \mathrm{~mm}$ e de M. bicolor dos $40 \mathrm{~mm}$. De acordo com Pereira et al. (2003) [7], esses tamanhos correspondem ao tamanho de comercialização para cada uma das espécies estudadas no estado de São Paulo, estando associado ao maior rendimento em carne. Christo \& Absher (2001) [33], analisando o índice de rendimento de $M$. charruana e $M$. guyanensis (=bicolor), na Baía de Paranaguá (Paraná), encontraram valores médios anuais de 50,9 e 43,4\% respectivamente. Os mesmos autores alegam que altos rendimentos mensais estão altamente ligados ao ciclo reprodutivo, quando as gônadas dos indivíduos estão altamente desenvolvidas, aumentando o peso de carne. Santos \& Christo (2010) [15] analisaram o rendimento médio de $M$. guyanensis (=bicolor) em duas populações naturais na Baía de Paranaguá (Paraná) e concluíram que a população associada a uma maior interferência antrópica obteve um rendimento médio de carne em torno de $30,8 \%$, enquanto que a população com menor interferência humana apresentou um rendimento de $44,5 \%$.

Para outros bivalves, observa-se rendimentos que variam de 9,5 a mais de 50,0\%. Yauri et al. (2011) [28] analisaram quatro espécies de bivalves explotadas na Ilha do Maranhão (MA) e concluíram que Macoma constricta e Tagelus plebeius obtiveram rendimentos superiores a 50\% quando comparadas com Protothaca antiqua e Anomalocardia brasiliana. Arrieche et al. (2002) [30] encontraram um rendimento de 24,8 a 31,8\% para o mexilhão Perna perna. Freitas (2011) [34], analisando o rendimento médio de Anomalocardia brasiliana, encontrou um valor de $9,5 \%$, porém com aumento para exemplares maiores. 
Tabela 1: Intervalos de confiança $(0,95)$ para os parâmetros " $a$ " $e$ " $b$ ” das relações morfométricas de M. charruana $e$ M. bicolor em Sergipe. Comprimento (CC), largura (LC) e altura (AC) da concha, $r^{2}=$ coeficiente de determinação, $N=$ tamanho da amostra, IC = intervalo de confiança, a = intercepto e $b=$ declividade.

\begin{tabular}{|c|c|c|c|c|c|c|c|c|c|}
\hline \multirow{2}{*}{$\begin{array}{c}\text { Relação } \\
\text { morfométrica }\end{array}$} & \multirow{2}{*}{ Espécie } & \multicolumn{2}{|c|}{ Parâmetros } & \multirow{2}{*}{$\mathbf{r}^{2}$} & \multirow{2}{*}{$\mathbf{N}$} & \multicolumn{2}{|c|}{ IC (a; 0,95) } & \multicolumn{2}{|c|}{ IC (b;0,95) } \\
\hline & & $\mathbf{a}$ & $\mathbf{b}$ & & & Inferior & Superior & Inferior & Superior \\
\hline \multirow{3}{*}{$\mathbf{L C}=\mathbf{a}+\mathbf{b C C}$} & M. charruana & 3,7965 & 0,3391 & 0,88 & 435 & 3,3124 & 4,2807 & 0,3270 & 0,3512 \\
\hline & & & & & & & & & \\
\hline & M. bicolor & 5,1308 & 0,3934 & 0,77 & 300 & 3,9447 & 6,3170 & 0,3684 & 0,4184 \\
\hline \multirow{3}{*}{$\mathbf{A C}=\mathbf{a}+\mathbf{b C C}$} & M. charruana & $-1,9163$ & 0,3546 & 0,82 & 435 & $-2,5453$ & $-1,2873$ & 0,3389 & 0,3706 \\
\hline & & & & & & & & & \\
\hline & M. bicolor & 1,1267 & 0,2977 & 0,70 & 300 & 0,0706 & 2,1827 & 0,2755 & 0,3200 \\
\hline \multirow{3}{*}{$\mathbf{P T}=\mathbf{a C C}$} & M. charruana & 0,0004 & 2,3976 & 0,86 & 435 & 0,0002 & 0,0008 & 2,2024 & 2,5928 \\
\hline & & & & & & & & & \\
\hline & M. bicolor & $8 \mathrm{E}-05$ & 2,8437 & 0,87 & 300 & $5 \mathrm{E}-05$ & $1,4 \mathrm{E}-06$ & 2,7175 & 2,9698 \\
\hline
\end{tabular}


Tabela 2: Rendimento médio de carne (\%) para M. charruana $e$ M. bicolor em diferentes classes de comprimento da concha (CC; $\mathrm{mm})$ em Sergipe.

\begin{tabular}{ccc}
\hline $\mathbf{C C}$ & \multicolumn{2}{c}{ Rendimento médio de carne $(\%)$} \\
\cline { 2 - 3 }$(\mathbf{m m})$ & M. charruana & M. bicolor \\
\hline $\mathbf{2 0}-\mathbf{3 5}$ & 52,2 & 31,6 \\
$\mathbf{3 5}-\mathbf{5 0}$ & 49,3 & 32,6 \\
$\mathbf{5 0}-\mathbf{6 5}$ & 50,5 & 34,5 \\
\hline
\end{tabular}

É interessante notar que, apesar do rendimento de carne de sutinga (M. charruana) ser superior ao do sururu de mangue (M. bicolor), o valor de venda por quilograma da sua carne ("catado") é $20 \%$ inferior (Sandra Lima dos Santos, observação pessoal). Essa diferença está associada ao modo de captura do sururu de mangue, o qual é coletado manualmente um a um, sendo um processo de extração mais difícil. Já o sutinga é coletado manualmente, porém vários exemplares são capturados ao mesmo tempo. Essas diferenças devem ser consideradas ao se considerar o manejo desse recurso pesqueiro.

\section{CONCLUSÃO}

Das duas espécies de Mytella (M. charruana e M. bicolor) capturadas no estado de Sergipe, a M. bicolor é a que atinge maiores tamanhos, porém com menor rendimento em carne. Porém, a adição dos dados apresentados nesse estudo para Sergipe não permitiu observar o padrão usualmente aceito de aumento de comprimento com o aumento da latitude.

O crescimento das duas espécies tanto em largura, altura e peso são do tipo alométrico negativo. Como os exemplares capturados correspondem ao tamanho normalmente explotado na região, sugere-se que um esforço seja feito, em futuros estudos, para analisar espécimes de menor tamanho a fim de avaliar alterações nas relações morfométricas da concha ao longo do crescimento do indivíduo, ou seja, possíveis mudanças na declividade das relações estimadas. A comparação dos parâmetros estimados nesse estudo com o de outras localidades e espécies exige uma padronização na metodologia adotada, o que atualmente não tem sido observado.

Programas contínuos de avaliação sazonal e anual dos parâmetros estimados, principalmente referentes ao rendimento em carne, devem ser mantidos a fim de que se possa fazer a conversão do catado em peso vivo total. Adicionalmente, deve-se fazer um esforço local para registrar a estatística pesqueira para essas duas espécies separadamente, o que atualmente não é realizado, considerando principalmente o rendimento de carne diferenciado entre as duas espécies.

\section{AGRADECIMENTOS}

Os autores agradecem aos(às) pescadores(as) de Nossa Senhora do Socorro e de Laranjeiras por terem realizado as coletas de sururu e sutinga, e a Isaac Trindade dos Santos por ter fornecido mapa com a rede hidrográfica de Sergipe.

\section{REFERÊNCIAS BIBLIOGRÁFICAS}

1. Benfield SL, Guzman HM, Mair JM. Temporal mangrove dynamics in relation to coastal development in Pacific Panama. Journal of Environmental Management. 2005; 76 (3): 263-276.

2. Boffi AV. Moluscos brasileiros de interesse médico e econômico. São Paulo. HCITEC; 1979. 182 p.

3. Casarini LM \& Henriques MB. Estimativa de estoque do mexilhão Perna perna e da espécie invasora Isognomon bicolor em bancos naturais da Baía de Santos, São Paulo, Brasil. Boletim do Instituto de Pesca. 2011; 37(1): 1-11.

4. Carriço JMM, Lopes FLG, Cruz AS, Nascimento IS. Identificação e proposição de um plano de manejo para os bancos de sururu do rio Sergipe. In: III Encontro Nacional dos Núcleos de Pesquisa Aplicada em Pesca e Aquicultura; 2011 Dez; Búzios (RJ). 
5. Bouchet P \& Huber, M (2012). Mytella charruana (d'Orbigny, 1842). In: MolluscaBase (2015). Accessed at http://www.molluscabase.org/aphia.php?p=taxdetails\&id=397146 on 2016-03-20.

6. Marques HL. A. Criação Comercial de Mexilhões. São Paulo. Nobel; 1998. 109 p.

7. Pereira OM, Hilberath RC, Ansarah PRAC, Galvão MSN. Estimativa da produção de Mytella falcata e de M. guyanensis em bancos naturais do estuário de Ilha Comprida-SP-Brasil. Boletim do Instituto de Pesca. 2003; 29(2): 139-149.

8. Sartori AF \& Rosenberg G (2016). Mytella guyanensis (Lamarck, 1819). In: MolluscaBase (2015). Accessed at http://molluscabase.org/aphia.php?p=taxdetails\&id=533145 on 2016-03-20

9. Araújo ARR, Silva FD, Santana RF, Lopes FC. Gestão da pesca de Mytella charruana (d'Orbigny, 1846) no litoral do estado de Sergipe: Indicadores de Sustentabilidade. Revista Brasileira de Engenharia de Pesca. 2009; 4(2): 56-70.

10. Instituto Brasileiro do Meio Ambiente e dos Recursos Naturais Renováveis. Estatística da pesca 2007 - Brasil: grandes regiões e unidades da federação / Brasília (DF): Ibama; 2007. 151 p.

11. Thomé-Souza MJF, Dantas Júnior JF, Silva FCB, Félix DCF, Santos JC. Estatística Pesqueira da Costa do Estado de Sergipe e Extremo Norte da Bahia - 2010. São Cristóvão: Editora UFS; 2012. 88 p.

12. Thomé-Souza MJF, Deda MS, Santos JP, Carvalho BLF, Araújo MLG, Filho EBG, Félix DCF, Santos JC. Estatística Pesqueira da Costa do Estado de Sergipe e Extremo Norte da Bahia - 2011. São Cristóvão: Editora UFS; 2013. 92 p.

13. Thomé-Souza MJF, Carvalho BLF, Silva CO, Deda MS, Filho EBG, Félix DCF, Santos JC. Estatística Pesqueira da Costa do Estado de Sergipe e Extremo Norte da Bahia - 2012. São Cristóvão: Editora UFS; 2014a. 102 p.

14. Thomé-Souza MJF, Carvalho BLF, Filho EBG, Silva CO, Deda MS, Félix DCF, Santos JC. Estatística Pesqueira da Costa do Estado de Sergipe e Extremo Norte da Bahia - 2013. São Cristóvão: Editora UFS; 2014b. 108 p.

15. Santos D \& Christo SW. Estudo populacional de Mytella guyanensis (Lamarck, 1819) em duas populações naturais do complexo estuarino da Baía de Paranaguá, Paraná - Brasil. In: Anais do XIX Encontro Anual de Iniciação Científica - UNICENTRO; 2010 Out. Guarapuava (PR).

16. Silva J, Farias Lins JL, Silva VM, Tavares AS, Araujo DM. Conchas de sururu Mytella charruana como possível ingrediente em dietas de peixes. In: I Encontro de Inovação, Tecnologia e Iniciação Científica do IFAL; 2014. Maceió (AL).

17. Gaspar MB, Santos MN, Vasconcelos P, Monteiro CC. Shell morphometric relationships of the most common bivalve species (Mollusca: Bivalvia) of the Algarve coast (southern Portugal). Hydrobiologia. 2002; 477: 73-80.

18. Granadeiro JP \& Silva MA. The use of otoliths and vertebrae in the identification and size-estimation of fish in predator-prey studies. Cybium. 2000; 24: 383-393.

19. Nishida AK \& Leonel RMV. Occurrence, population dynamics and habitat characterization of Mytella guyanensis (Lamark, 1819) (Mollusca, Bivalvia) in the Paraíba do Norte river esturary. Boletim do Instituto Oceanográfico. 1995; 43(1): 41-49.

20. Cruz, RA \& Villalobos CR. Shell length at sexual maturity and spawning cycle of Mytella guyanensis (Bivalvia: Mytilidae) from Costa Rica. Revista de Biologia Tropical. 1993; 41(1): 89-92.

21. Rios EC. Seashells of Brazil. Rio Grande: Fundação Cidade do Rio Grande, Fundação Universidade do Rio Grande, Museu Oceanográfico; 1985. 329 p.

22. Pereira OM, Galvão MSN, Pimentel CM, Henriques MB, Machado IC. Distribuição dos bancos naturais e estimativa de estoque do gênero Mytella no estuário de Cananéia, SP, Brasil. Brazilian Journal of Aquatic Science and Technology. 2007; 11(1): 21-29.

23. Carpes-Paternoster S. Ciclo reprodutivo do marisco-do-mangue Mytella guyanensis (Lamark, 1819) no manguezal do Rio Tavares - Ilha de Santa Catarina/SC [dissertação]. Florianópolis (SC): Universidade Federal de Santa Cantarina; 2003. 40 p.

24. Henriques MB, Marques HLA, Pereira OM, Bastos GCC. Aspectos da estrutura populacional do mexilhão Perna perna, relacionados à extração em bancos naturais da Baía de Santos, estado de São Paulo, Brasil. Boletim do Instituto de Pesca. 2004; 30(2): 117-126.

25. Santos HSS, Beasley CR, Tagliaro CH. Changes in population characteristics of Mytella falcata (d'Orbigny, 1846) beds, an exploited tropical estuarine mussel. Boletim do Instituto de Pesca. 2010; 36(2): 85-97.

26. Katsanevakis S, Thessalou-Legaki M, Karlou-Riga C, Lefkaditou E, Dimitriou E, Verriopoulos G. Information-theory approach to allometric growth of marine organisms. Marine Biology. 2006; 151(3): 949-960.

27. Froese R. Cube law, condition factor and weight-length relationships: history, meta-analysis and recommendations. Journal of Applied Ichthyology. 2006; 22: 241-253. doi:10.1111/j.14390426.2006.00805.x. 
28. Yauri WML, Azevedo MCS, Mendes DGS, Oliveira KM, Silva JS. Biometria e fator de condição dos moluscos arenícolas dominantes no estuário do Rio Paciência no município da Raposa/MA. In: X Congresso de Ecologia do Brasil; 2011 Set. São Lourenco (MG).

29. Schaeffer-Novelli Y. Análise populacional de Anomalocardia brasiliana (Gmelin, 1791), na Praia do Saco da Ribeira, Ubatuba, estado de São Paulo. Boletim do Instituto Oceanográfico. 1980; 29(2):351355.

30. Arrieche D, Licet B, García N, Lodeiros C, Prieto A. Índice de condición, gonádico y de rendimiento del mejillón marrón Perna perna (Bivalvia: Mytilidae), Del Morro de Guarapo, Venezuela. Interciência. 2002; 27(11): 613-619.

31. Souza AB. Relações alométricas da Anomalocardia brasiliana na praia de Mangue Seco, PernambucoBrasil [dissertação]. Recife (PE): Universidade Federal Rural de Pernambuco; 2012. 46 p.

32. Santos JJS, Terceiro AM, Yauri WLM. Dinâmica da população de Anomalocardia brasiliana (Mollusca, Bivalvia, Veneridae) no estuário do Rio Paciência, no município da Raposa, estado do Maranhão. Anuário do Instituto de Geociências - UFRJ. 2014; 37(1): 61-69.

33. Christo SW \& Absher TM. Ciclo reprodutivo de Mytella guyanensis e Mytella charruana (Bivalvia: Mitilidae) na Baía de Paranaguá, Paraná. In: IX Congresso Latino-Americano sobre Ciências del Mar; 2001 Set; San André Isla (Colombia).

34. Freitas ST. Anomalocardia brasiliana Gmelin, 1791 (Mollusca Bivalvia): rendimento, composição química e dados etnobiológicos das marisqueiras de Barra Grande, Cajueiro da Praia, Piauí [dissertação]. Teresina (PI): Universidade Federal do Piauí; 2011. 96 p. 